\title{
A Recessive Resistance to Rice yellow mottle virus Is Associated with a Rice Homolog of the CPR5 Gene, a Regulator of Active Defense Mechanisms
}

\author{
Julie Orjuela, ${ }^{1}$ E. F. Thiémélé Deless, ${ }^{2}$ Olufisayo Kolade,${ }^{3}$ Sophie Chéron, ${ }^{1}$ Alain Ghesquière, ${ }^{1}$ and \\ Laurence Albar ${ }^{1}$ \\ ${ }^{1}$ IRD, UMR DIADE, F-34394 Montpellier 5, France, ${ }^{2}$ CNRA, Bimbresso, 01 BP 1536 Abidjan 01, Côte d'Ivoire, ${ }^{3}$ Africa Rice \\ Center, 01 BP 2031, Cotonou, Benin
}

Submitted 3 May 2013. Accepted 29 July 2013.

\begin{abstract}
$R Y M V 2$ is a major recessive resistance gene identified in cultivated African rice (Oryza glaberrima) which confers high resistance to the Rice yellow mottle virus (RYMV). We mapped $R Y M V 2$ in an approximately $30-\mathrm{kb}$ interval in which four genes have been annotated. Sequencing of the candidate region in the resistant Tog7291 accession revealed a single mutation affecting a predicted gene, as compared with the RYMV-susceptible $\boldsymbol{O}$. glaberrima CG14 reference sequence. This mutation was found to be a one-base deletion leading to a truncated and probably nonfunctional protein. It affected a gene homologous to the Arabidopsis thaliana CPR5 gene, known to be a defense mechanism regulator. Only seven $O$. glaberrima accessions showing this deletion were identified in a collection consisting of 417 accessions from three rice species. All seven accessions were resistant to RYMV, which is an additional argument in favor of the involvement of the deletion in resistance. In addition, fine mapping of a resistance quantitative trait locus in $O$. sativa advanced backcrossed lines pinpointed a 151-kb interval containing RYMV2, suggesting that allelic variants of the same gene may control both high and partial resistance.
\end{abstract}

The use of natural host-derived resistance $(R)$ genes is one of the best methods to control virus-induced disease. Khetarpal and associates (1998) listed 139 virus $R$ genes; however, the nature of only a few of them is known. Most belong to two main types of genes which are effective in various plant-virus interactions. The first type corresponds to nucleotide-binding leucine-rich repeat (NB-LRR) genes, resulting in dominant resistance and whose role is ubiquitous in plant-pathogen interactions (Dodds and Rathjen 2010). They are associated with the development of active defense mechanisms. At least six NB-LRR genes, including $N, R x$, and $S w-5$, have been reported

Nucleotide sequence data is available under the following accession numbers: sequence of the $30 \mathrm{~kb}$ containing RYMV2 in Tog7291, KC977156; genomic sequence of LOC_Os01g68970 in the Azucena and IR64 accessions, KC977153 and KC977154, respectively; partial CDS corresponding to LOC_Os01g68970.1 gene model in the Azucena, IR64, and CG14 accessions, KC977151, KC977152, and KF303596, respectively.

Corresponding author: L. Albar; E-mail: laurence.albar@ird.fr

* The $\boldsymbol{e}$-Xtra logo stands for "electronic extra" and indicates that a supplementary figure is published online.

(C) 2013 The American Phytopathological Society to be involved in virus resistance (Cournoyer and Dineskumar 2011). The second type corresponds to translation initiation factors. Because of their small genomes, viruses rely on host cellular factors to achieve their infectious cycle. The absence of such factors or mutations in them results in impaired susceptibility, which is generally recessive (Gomez et al. 2009). Translation initiation factors are the most documented $R$ genes falling in this category. Both eIF4E and eIF4G, or their isoforms, are responsible for resistance to viruses of different families in diverse plant species (Le Gall et al. 2011). In addition to these two main types of virus $R$ genes, other natural $R$ genes from very diverse gene families have been described. However, thus far, they have only proved efficient in a single or small number of pathosystems. For instance, the RTM gene complex (Cosson et al. 2010) is effective against the Tobacco etch virus, Lettuce mosaic virus, and Plum pox virus (Decroocq et al. 2006) but has only been detected in Arabidopsis thaliana. The $R$ genes identified so far probably constitute the tip of the iceberg among the genes naturally involved in virus resistance. Such candidate genes may be involved in silencing, which is a primary anti-viral response (Mlotshwa et al. 2008); in defense mechanisms (Cournoyer and Dinesh-Kumar 2011); or in direct interactions with the viral components (Brizard et al. 2006; Di Carli et al. 2010).

Rice is both a common staple worldwide and one of the most studied plants due to the comparative advantages of its small genome. In all rice-growing areas, viruses have been described as major pathogens that can drastically reduce crop yields (Siré and Brugidou 2002). However, few $R$ genes against rice viruses have been cloned to date (Albar et al. 2006; Lee et al. 2010; Yoshii et al. 2009). One of the reasons may be that some of the most damaging viruses on rice, such as Tungro viruses, are only transmitted by insects, which dramatically complicates the characterization of resistance. The Rice yellow mottle virus (RYMV) is endemic to Africa and responsible for major yield losses in irrigated conditions (Kouassi et al. 2005). Because RYMV is highly infectious and mechanically transmissible, it is a powerful model for studying rice-virus interactions. Both partial and high resistances against RYMV have been described. In Asian cultivated rice (Oryza sativa), partial resistance is generally associated with japonica varieties, and quantitative train loci (QTL) of partial resistance have been mapped (Albar et al. 1998). In particular, a major QTL, hereafter referred to as RYMV-QTL1, was located on chromosome 1 in an 11-centimorgan (cM) confidence interval (Boisnard et al. 2007). High resistance is very uncommon in $O$. sativa - a single major gene $(R Y M V l)$ that encodes an 
eIF(iso)4G factor has been described thus far (Albar et al. 2006). In contrast, high resistance appears to be more frequent in African cultivated rice (O. glaberrima), in which a second recessive $R$ gene ( $R Y M V 2$ ) has been identified (Thiémélé et al. 2010). In this article, we describe the fine mapping of this gene. The sequencing of the target region in the resistant parent and an analysis of variability in a collection of accessions pinpointed a gene whose homolog in A. thaliana is involved in defense mechanisms. Thus, RYMV2 and $R Y M V 1$ appeared to control resistance through completely different pathways. In parallel, fine mapping of RYMV-QTL1 using advanced backcross (BC) lines revealed its colocalization with $R Y M V 2$ and suggested that the same gene could be involved in high and partial resistance.

\section{RESULTS}

\section{Mapping of the RYMV2 major $R$ gene.}

The first steps of $R Y M V 2$ mapping involved an $O$. glaberrima intraspecific F2 population of 156 informative plants derived from a cross between the Tog7291 accession, homozygous for a resistance allele on $R Y M V 2$, and Tog5681, homozygous for a susceptibility allele on that gene. In all, 107 susceptible and 49 resistant plants were observed, which fitted a 3:1 susceptible/resistant segregation pattern $\left(\chi^{2}=3.42 ; P>0.05\right)$, indicating monogenic and recessive inheritance of Tog7291 resistance, as previously reported (Thiémélé et al. 2010). A subset of 18 resistant plants was genotyped with markers located in candidate regions (i.e., regions where QTL of partial resistance or genes from $e I F 4 E$ and $e I F 4 G$ families-known to be involved in virus resistance-had previously been identified) (Boisnard et al. 2007). Markers located on chromosome 1 close to the $I F 4 E$ gene and RYMV-QTL1 showed clear distortion of segregation toward the Tog7291 allele (17 plants with the Tog7291 allele for marker RM8236), suggesting that this region is associated with resistance. This was confirmed using the whole intraspecific mapping population. RYMV2 was mapped between RM12055 and RM8236 markers that flanked a 514-kb interval on the reference sequence of the Nipponbare accession. At this step, the $e I F 4 E$ gene mapped outside the target interval and was rejected as a putative candidate.

Because the low level of polymorphism impaired $R Y M V 2$ fine mapping in an $O$. glaberrima intraspecific population, interspecific mapping populations were derived from ([IR64 $\times$ Tog7291] $\times$ Tog7291) crosses that involved the susceptible $O$. sativa IR64 accession. In total, $43 \mathrm{BC} 1 \mathrm{~F} 1,318 \mathrm{BC} 1 \mathrm{~F} 2$, and 3,470 BC1F3 plants were genotyped using markers flanking the candidate region. Sixty-four recombinant plants were phenotyped for RYMV resistance and genotyped using additional markers from the candidate region. The RYMV2 locus was narrowed down to the interval defined by the RM12083 and ID272679 markers (Fig. 1A).

\section{Sequence analysis of the $R Y M V 2$ region.}

In the $O$. sativa subsp. japonica Nipponbare public sequence (IRGSP-1.0), the RM12083-ID272679 interval containing the

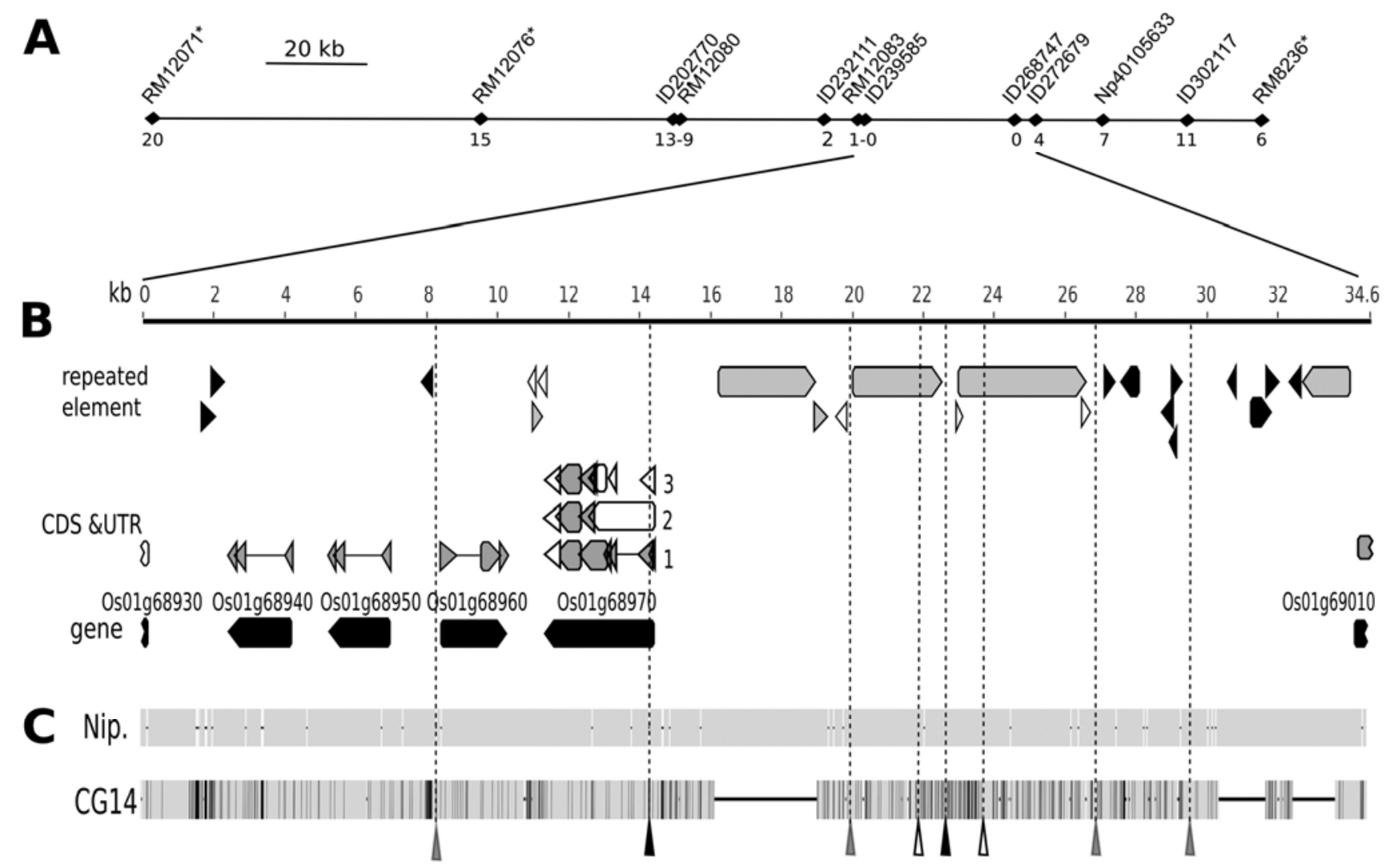

Fig. 1. A, Physical map of RYMV2 region. The number of recombinants between RYMV2 and each marker in the interspecific population is indicated above the marker names. Recombinants resulted from a screening of approximately 1,800 plants for markers indicated with an asterisk $(*)$ and 3,831 plants for other markers. B, Annotation of the RM12083-ID272679 interval on the Nipponbare sequence. Gene models are from the Michigan State University Rice Genome Annotation Project (v7.0); annotations of repeated elements are from Rice Annotation Project Database (RAP-DB). Gene loci are represented in black and gene models are shown in gray for coding sequences and white for untranslated regions (UTR). Among the repeated elements, miniature invertedrepeat transposable elements are represented in black, retrotransposons in gray, and others in white. C, Alignment between Nipponbare and CG14 sequences on the RM12083-ID272679 interval. Deletions are represented as horizontal black lines. Vertical black lines in the CG14 sequence represent polymorphism with the Nipponbare sequence. Confirmed single-nucleotide polymorphisms (SNPs) between CG14 and Tog7291 are indicated by black arrows; putative SNPs or differences in homopolymer size are indicated by empty or gray arrows, respectively. 
$R Y M V 2 R$ gene corresponds to a stretch of $34.6 \mathrm{~kb}$ between coordinates 40,058,342 and 40,092,935 bp on chromosome 1 . In this interval, the Michigan State University (MSU) Rice Annotation Project (v7.0) reports gene annotations LOC Os01g68940, LOC_Os01g68950, LOC_Os01g68960, LOC_ Os01g68970, the first $267 \mathrm{bp}$ of the LOC_Os01g69010 gene, and $63 \mathrm{bp}$ of the $5^{\prime}$ untranslated region (UTR) of the LOC Os01g68930 gene (Fig. 1B; Table 1). The four complete predicted genes were located in the first proximal $15 \mathrm{~kb}$ of the interval, while the distal side of the interval contained repeated elements, essentially including miniature inverted-repeat transposable elements and retrotransposons. A single gene model is proposed for all genes except LOC_Os01g68970, for which three different models are proposed. The LOC_Os01g68970.1 gene model predicted a protein of 546 amino acids while the LOC_Os01g68970.2 and LOC_Os01g68970.3 gene models predicted the same 273-amino-acid protein (Fig. 2A).

In the $O$. glaberrima CG14 sequence, the length of the RM12083-ID272679 interval is only $30.1 \mathrm{~kb}$ between coordinates 286,406 and 316,460 within contig010305. Comparisons between CG14 and Nipponbare sequences in the RYMV2 region revealed the presence of stretches of conserved segments interrupted by three major indels of 1.2 to $3 \mathrm{~kb}$, which explains the difference in size of the target interval between the two accessions (Fig. 1C). These three indels concern the distal side of the interval containing repeated elements. Point mutations and short indels were observed in the gene-rich region. However, the percentages of identity on the genes and predicted proteins completely included in the interval were above $95 \%$ (Table 1), and no drastic mutation resulting in a frameshift or a STOP codon in the coding sequence (CDS) or affecting the splicing sites was noted, suggesting that the gene models predicted in Nipponbare must be reliable in CG14.

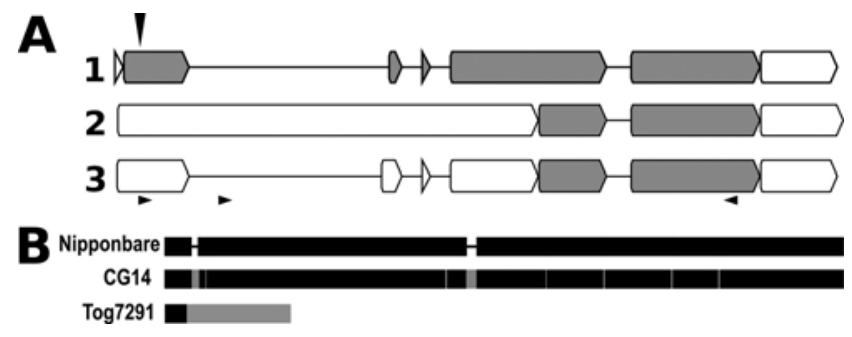

Fig. 2. A, Comparison of the three gene models corresponding to $\mathrm{LOC}_{-}$ Os01g68970 locus. Coding sequences are represented by gray boxes, $3^{\prime}$ and $5^{\prime}$ untranslated regions (UTR) by white boxes, and introns by horizontal lines. A vertical black arrow above gene model 1 indicates the position of the one-base deletion characterizing Tog7291 accession. Small horizontal black arrows below gene model 3 represent the primers used to validate experimentally the different gene models. B, Comparison of the proteins corresponding to LOC_Os01g68970.1 in Oryza sativa Nipponbare and $O$. glaberrima CG14 and Tog7291 accessions. Horizontal lines represent deletions; gray boxes represent polymorphisms between $O$. glaberrima and Nipponbare sequences.
The RM12083-ID272679 interval was sequenced using a Sanger sequencing approach on polymerase chain reaction (PCR) fragments in O. glaberrima Tog7291, which carries a resistance allele on $R Y M V 2$. The Tog7291 sequence was compared with the RYMV-susceptible CG14 sequence. Inconsistent 1- to 2-bp size differences of four homopolymers were obtained across several sequencing repeats. Similarly, two inconsistent nucleotides on or between highly repeated elements were observed. Therefore, these sites were considered as putatively polymorphic between Tog7291 and CG14. Finally, this analysis revealed two confirmed and six putative singlenucleotide polymorphisms (SNPs) or short indels between Tog7291 and CG14. An SNP was confirmed in a repeated sequence and a one-base deletion was confirmed in the LOC Os01g68970 gene (Fig. 1C). According to the LOC_ Os01g68970.2 and LOC_Os01g68970.3 gene models, this one-base deletion in Tog7291 is located in the 5' UTR (Fig. 2A). According to the LOC_Os01g68970.1 gene model, this deletion is located in the first exon of the CDS and results in a frameshift from the 18th amino acid and in a STOP codon a few amino acids downstream (Fig. 2B). In this case, Tog7291 would possess a null allele at LOC_Os01g68970 locus.

To characterize the transcripts of the LOC_Os01g68970 gene, cDNA amplification products were obtained from $O$. sativa IR64 and Azucena accessions and O. glaberrima CG14 accession using gene-specific primers. Amplification product sizes were in agreement with the LOC_Os01g68970.1 and LOC_Os01g68970.3 gene models, whereas no amplification corresponding to the LOC_Os01g68970.2 gene model was observed. The amplification products were cloned and two different clones were sequenced for each accession. The six sequences obtained were in agreement with the LOC_ Os01g68970.1 gene model. In addition, the protein corresponding to the LOC_Os01g68970.1 gene model presented higher similarity with a homologous protein identified in various eudicot or monocot species, whereas the protein predicted by the LOC_Os01g68970.2 and LOC_Os01g68970.3 gene models lacked part of the conserved regions identified in the other species (Supplementary Fig. 1). This suggests that a functional protein corresponding to the LOC_Os01g68970.1 gene model must be produced in rice and, consequently, that Tog7291 must have a null allele.

\section{Exploration of the diversity in the RYMV2 region in a collection of accessions.}

The two confirmed mutations that distinguished Tog7291 from CG14 sequences in the RYMV2 region were associated with polymorphism on $C f o$ I restriction sites. Cleaved amplified polymorphic sequence (CAPS) markers targeting these mutations were developed. CAPS1 targeted the one-base deletion in the LOC_Os01g68970 gene. CAPS2 targeted the SNP identified in the repeated region. CAPS1 and CAPS2 were used to screen a collection of 417 rice accessions representing the two cultivated species $O$. sativa and $O$. glaberrima and the African wild

Table 1. Characteristics of the genes annotated in the RM12083-ID272679 interval containing RYMV2, according to the MSU Rice Genome Annotation Project $^{\mathrm{a}}$

\begin{tabular}{llccc}
\hline Gene model & \multicolumn{1}{c}{ Product } & Transcript evidence & Nucleotide ID (\%) & Amino acid ID (\%) \\
\hline LOC_Os01g68930 & Expressed protein & Yes & 90.3 & $\ldots$ \\
LOC_Os01g68940 & Ubiquitin family domain containing protein & Yes & 95.4 & 100 \\
LOC_Os01g68950 & Ubiquitin family domain containing protein & Yes & 99.5 & 100 \\
LOC_Os01g68960 & Conserved hypothetical protein & No & 99.2 & 98.5 \\
LOC_Os01g68970 & HYS1, putative & Yes & 98.3 & 96.8 \\
LOC_Os01g69010 & MATE efflux protein, putative & No & 92.0 & 91.7 \\
\hline
\end{tabular}

${ }^{a}$ Transcript evidence refers to the cDNA or expressed sequence tag reported on the Rice Genome Annotation Project website. The percentage of identity at nucleotide and amino acid levels between CG14 and 'Nipponbare' was estimated based on the alignment of the CG14 sequence on the Nipponbare gene models. Only the 63 and 267 bp included in the interval containing RYMV2 were considered for LOC_Os01g68930 and LOC_Os01g69010, respectively. 
species $O$. barthii. This collection was also screened, based on the Illumina VeraCode technology, with an SNP located in the second intron of the LOC_Os01g68970 candidate gene (J. Orjuela, unpublished data). This SNP, hereafter referred to as SNP3, was not polymorphic between CG14 and Tog7291.

Four different haplotypes were observed (Table 2). All $O$. sativa accessions shared the same haplotype, which was also the main haplotype among $O$. barthii accessions. Two additional haplotypes were present in $O$. barthii but one of them was found in a single accession. The three haplotypes observed in $O$. barthii were also found in $O$. glaberrima but in quite different proportions. A fourth haplotype was specific to $O$. glaberrima accessions. The choice of SNPs selected to be polymorphic between $O$. glaberrima accessions explains the apparent higher level of diversity observed in African rice compared with $O$. sativa, which is contradictory to the known diversity levels between the two cultivated rice species. The haplotype present only in $O$. glaberrima species was characterized by a rare allele on the CAPS1 marker; this allele was only present in $2.7 \%$ of $O$. glaberrima accessions and absent in other species. The seven accessions showing this allele were Tog10434, Tog6698, IG38, IG35, IRGC103549, and IRGC112577, in addition to $\operatorname{Tog} 7291$. These seven accessions were evaluated for RYMV resistance based on symptom observation on eight plants per accession and on virus content estimated with an enzyme-linked immunosorbent assay (ELISA) test on four plants per accession. None of these accessions showed symptoms or detectable virus content (Table 3), which confirmed the results previously obtained by Thiemele and associates (2010) on five of them. Thus, these seven accessions were considered to be highly resistant. The genotype-resistance association was tested using a Fisher exact test, considering only accessions for which the phenotype was available and without any known resistance allele on $R Y M V 1$. The association was found to be nonsignificant for SNP3 $(P=1)$ and CAPS2 $(P=$ $0.74)$ markers and highly significant for CAPS1 $\left(P=3 \times 10^{-8}\right)$.

\section{Fine mapping of RYMV-QTL1.}

Genetic mapping of RYMV partial resistance previously identified the RYMV-QTL1 as being one of the main QTL in the IR64 $\times$ Azucena population (Albar et al. 1998). According to Boisnard and associates (2007), this QTL is located in a 2.3-Mb interval flanked by RM431 and RM5310 markers on chromosome 1. Here, BC3F2 plants derived from BC3F1 heterozygous for the RM431-RM5310 interval were screened for recombination between these two markers. Recombinants were further genotyped with additional simple-sequence repeat (SSR) or indel markers of the candidate region in order to refine the positions of recombination events. For each recombinant, up to four recombinant $\mathrm{BC} 3 \mathrm{~F} 3$ progenies, homozygous on both sides of the recombination, were selfed, and the resulting BC3F4 progenies were phenotyped. Similarly, additional recombinants were identified among $\mathrm{BC} 3 \mathrm{~F} 3$ plants derived from $\mathrm{BC} 3 \mathrm{~F} 2$ plants heterozygous at RYMV-QTL1. In total, 17 recombinants were identified and characterized (Fig. 3A).
A first phenotyping step involved the 17 available recombinant families. The symptom intensity score was 4.15 to 5.26 (Fig. 3B) and the heritability of the traits was 0.58 . The symptom intensity score distribution suggested a bimodal distribution pattern (Fig. 3F). A Student's $t$ test was performed to analyze the effect of the genotype at each individual marker on the symptom intensity score (Fig. 3E). Markers included in a 282 $\mathrm{kb}$ interval between the RM12076 and RM12092 markers were strongly associated with the symptom intensity score $(t>$ $20 ; P<0.005)$, with the Azucena allele increasing the resistance level, thus suggesting that RYMV-QTL1 is located in this interval. However, the heterogeneity of resistance among progenies with the Azucena allele in the RM12076-RM12092 interval suggested that additional QTL might segregate in these families. This result is in agreement with the high number of QTL for partial resistance identified in the IR64 $\times$ Azucena population (Boisnard et al. 2007).

In a second phenotyping experiment, we focused on the progenies derived from the $\mathrm{BC} 3 \mathrm{~F} 2-240$ plant, which was recombined between ID303300 and RM8236 (Fig. 3C and 3D). Progenies fixed for the Azucena allele before the recombination event were significantly more resistant than those fixed for the IR64 allele throughout the region $(t=12 ; P=0.0012)$. This suggested that the QTL was located before the recombination event (i.e., before RM8236) and pinpointed a 151-kb region delimited by RM12076 and RM8236 markers (interval $39,991,994$ to $40,142,999$ on the IRGSP-1.0 chromosome 1 pseudomolecule).

In total, 17 genes, including all the candidate genes for RYMV2, particularly LOC_Os01g68970, were annotated in the RM12076-RM8236 interval. LOC_Os01g68970 was sequenced in IR64 and Azucena to identify putative polymorphisms be-

Table 3. Phenotypic characterization of the seven accessions showing B genotype on cleaved amplified polymorphic sequence (CAPS) 1 marker $^{\mathrm{a}}$

\begin{tabular}{lccc}
\hline Accessions & CAPS1 & Symptoms & \multicolumn{1}{c}{ DO } \\
\hline Tog10434 & B & R & $0.065 \pm 0.001$ \\
Tog6698 & B & R & $0.065 \pm 0.001$ \\
IG38 & B & R & $0.065 \pm 0.001$ \\
IG35 & B & R & $0.066 \pm 0.001$ \\
IRGC103549 & B & R & $0.065 \pm 0$ \\
IRGC112577 & B & R & $0.065 \pm 0.001$ \\
Tog7291 & B & R & $0.065 \pm 0.001$ \\
Tog6208 & A & S & $1.088 \pm 0.023$ \\
Tog7020 & A & S & $1.072 \pm 0.035$ \\
IR64 & A & S & $1.070 \pm 0.042$ \\
Nipponbare & A & S & $1.012 \pm 0.011$ \\
Azucena & A & I & $0.247 \pm 0.067$ \\
Buffer & $\ldots$ & $\ldots$ & $0.065 \pm 0.001$ \\
\hline
\end{tabular}

a The Oryza glaberrima Tog6208 and Tog7020 accessions and the O. sativa IR64, Nipponbare and Azucena were used as controls. Symptoms were observed on eight plants per accession 2 weeks after inoculation and were noted S, I, and R when strong, intermediate, or no symptoms, respectively, were observed. DO represents the mean and the standard deviation resulting from individual enzyme-linked immunosorbent assay tests on four plants per accession, 9 days after inoculation.

Table 2. Haplotype diversity at the RYMV2 locus in three rice species

\begin{tabular}{|c|c|c|c|c|c|c|c|}
\hline \multicolumn{3}{|c|}{ Markers $^{\mathrm{a}}$} & \multirow[b]{2}{*}{ O. sativa } & \multirow[b]{2}{*}{ O. barthii } & \multicolumn{3}{|c|}{ Oryza $_{\text {glaberrima }}{ }^{\mathbf{b}}$} \\
\hline SNP3 & CAPS1 & CAPS2 & & & Total & $\mathbf{R}$ & $\mathbf{S}$ \\
\hline A & $\mathrm{A}$ & B & 56 & 79 & 8 & 0 & 1 \\
\hline B & A & A & 0 & 1 & 39 & 2 & 29 \\
\hline B & A & B & 0 & 20 & 207 & 7 & 110 \\
\hline B & B & B & 0 & 0 & 7 & 7 & 0 \\
\hline
\end{tabular}

${ }^{\text {a }} \mathrm{SNP}=$ single-nucleotide polymorphism and CAPS = cleaved amplified polymorphic sequence.

${ }^{\mathrm{b}}$ For $O$. glaberrima accessions, in addition to the total number of accessions, the number of accessions evaluated as susceptible (S) and resistant (R) to Rice yellow mottle virus (RYMV) without a resistance allele on the RYMVI gene are indicated. 
tween the two varieties. At the protein level, the two varieties were highly similar, with $98.5 \%$ identity and eight amino-acid substitutions. At the nucleotide level, IR64 and Azucena shared $99.6 \%$ identity between the ATG and STOP codons in the genomic DNA sequence but only $86.6 \%$ identity for the 700 nucleotides upstream of the ATG codon. The main differences in the promotor and $5^{\prime}$-UTR region concerned a 32-bp deletion in IR64 and a 49-bp deletion in Azucena (and in Nipponbare) compared with the CG14 sequence. These two deletions concerned flanking but nonoverlapping fragments located approximately 300 bp before the ATG codon.

\section{DISCUSSION}

A fine-mapping analysis pinpointed an interval of approximately $30 \mathrm{~kb}$ containing the $R Y M V 2 R$ gene. Sequence comparison between accessions carrying a resistance versus a susceptibility allele on RYMV2 (Tog7291 versus CG14) identified only two confirmed and six putative polymorphic sites. This extremely low intra- $O$. glaberrima polymorphism level war- ranted the sequencing of a several-kilobyte candidate region for the identification of polymorphism involved in expression of the trait of interest. Most of the polymorphisms concerned a repeated element-rich region. Nongenic region cannot be a priori excluded as determinants of a major phenotype but the most interesting polymorphism is a putative loss-of-function mutation in gene LOC_Os01g68970, whose homolog in A. thaliana is involved in constitutive resistance. This very uncommon polymorphism was associated with resistance among a collection of rice accessions and was found to be present in only seven $O$. glaberrima accessions. All of these accessions were highly resistant to RYMV but they originated from different countries (Liberia, Côte d'Ivoire, Mali, and Burkina Faso) and differed at the phenotypic level (data not shown), which confirmed that they represent distinct genotypes. These accessions showed the most frequent alleles in the O. glaberrima species for the two other flanking SNPs. This suggested that the local haplotype predating the one-base deletion was relatively common in $O$. glaberrima and that these accessions do not present any other rare polymorphism which could be
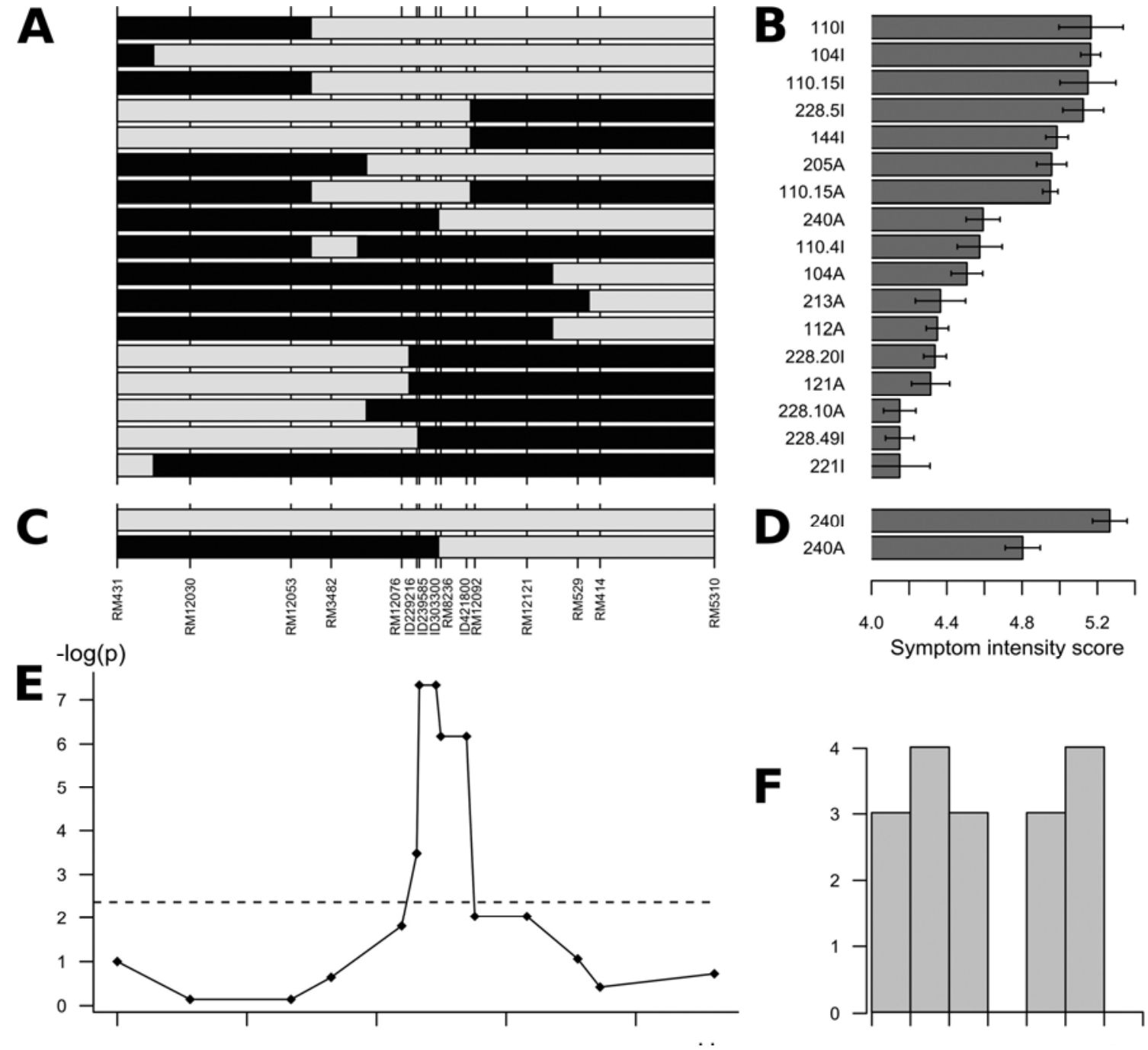

Fig. 3. A, Graphical genotype of families obtained after selfing of the recombinant plants used for RYMV-QTL1 fine mapping. Black segments represent alleles of the Azucena partially resistant parent; gray segments represent alleles of the IR64 susceptible parent. B, Symptom intensity score obtained for the progenies of each recombinant family. Error bars represent standard error. C, Graphical genotype of both types of BC2F3 progenies derived from the BC3F2-240 recombinant and fixed on both sides of the recombination. D, Symptom severity score obtained for BC3F2-240 progenies. For A through D, genotype and phenotype of a same progeny are represented on the same line, with the name of the progeny indicated between the genotype and phenotype. E, Probabilities (represented as $-\log [\mathrm{p}]$ ), estimated by a Student's $t$ test, that the symptom intensity score is associated to genotype at each marker. The dotted line corresponds to a probability of $P=0.005(-\log [\mathrm{p}]=2.3)$. In $\mathrm{A}, \mathrm{C}$, and $\mathrm{E}$, a same marker corresponds to the same vertical line. F, Distribution of the symptom intensity score in the progenies of recombinant families represented in A and B. 
associated with resistance. Finally, the few polymorphisms observed between CG14 and Tog7291 in the candidate region, the role of the A. thaliana homolog of LOC_Os01g68970 in defense mechanisms, and the association of CAPS1 polymorphism with the resistance level in a collection of $O$. glaberrima accessions constitute a body of evidence which supports the role of the one-base deletion in LOC_Os01g68970 in Tog7291 resistance against RYMV. The identification of accessions that had neither a known resistance allele on RYMV1 nor the one-base deletion in LOC_Os01g68970 suggest that other RYMV resistance factors are still to be discovered in $O$. glaberrima.

LOC_Os01g68970 was homologous to the At5g6493 gene, which is also called constitutive expresser of pathogenesisrelated genes-5 (CPR5). The corresponding protein contains five putative transmembrane domains (Yoshida et al. 2002). A. thaliana cpr 5 mutants show constitutive resistance to bacterial and oomycete pathogens (Bowling et al. 1997), and this resistance is associated with increased salicylic acid levels and constitutive expression of the pathogenesis-related 1 gene. The effect of $C P R 5$ on virus resistance appears to be contrasted. A. thaliana cpr5 mutants express enhanced resistance to the Cauliflower mosaic virus (Love et al. 2007), the Pepper mild mottle virus, and the Tobacco mild green mosaic virus (Fujisaki et al. 2009). In contrast, multiplication of the Spring beauty latent virus is not affected by the cpr5-2 mutation, and the Brome mosaic virus even shows increased multiplication in the cpr5-2 mutant, while A. thaliana is considered to be a nonhost species of this virus. The pleiotropic effect of CPR5 may explain these contrasted effects on plant-virus interactions. Indeed, in addition to its involvement in defense mechanisms, CPR5 has been reported to have an effect on cell proliferation and cell death control (Kirik et al. 2001), wall biogenesis and trichome development (Brininstool et al. 2008), response to sugars (Yoshida et al. 2002), and senescence regulation (Jing et al. 2002). Based on an analysis of double mutants, Jing and Dijkwel (2008) suggested that CPR5 may independently control multiple cellular processes and proposed that $C P R 5$ may be a master regulator of the cellular reactive oxygen species status or signaling. A single copy of CPR5 is present in A. thaliana and in additional eudicots, according to GreenPhylDB (Rouard et al. 2011). Conversely, two copies of $C P R 5$ are present in O. sativa, Zea mays, Sorghum bicolor, and Brachypodium distachyon. This suggests a more complex regulation of cellular processes controlled by CPR5 in monocots compared with eudicots.

Recessive $R$ genes, whose frequency is high in plant-virus interactions compared with plant-pathogen interactions in general, may be considered as susceptibility genes. The $R$ gene products may be required by pathogens for their growth in parasitized plants or may be negative regulators of plant defense responses (Pavan et al. 2009), like the mlo gene in barley (Büschges et al. 1997). Most recessive $R$ genes against plant viruses identified thus far belong to the translation initiation factor family and encode eIF4E, eIF4G, or their isoforms. $R Y M V 1$, for instance, confers resistance to RYMV through mutations in the eIF(iso) $4 \mathrm{G}$ factor (Albar et al. 2006). Although the role of these genes in plant-virus interactions is not precisely understood (Wang and Krishnaswamy 2012), they encode proteins whose direct interaction with a viral factor is necessary for the virus to accomplish its cycle. In contrast, according to the CPR5 gene function in A. thaliana, RYMV2 presumably corresponds to a negative regulator of the defense response, showing a null allele in highly resistant accessions. Thus, Tog7291 resistance would be associated with an active defense mechanism, which was not expected for a recessive resistance but is reminiscent of the induction of defense response genes observed by Gonzales-Ibeas and associates (2012) in the reces- sive resistance to the Watermelon mosaic virus. Thus, the two types of susceptibility factor described by Pavan and associates (2009) have been selected independently in $O$. glaberrima to control RYMV resistance.

Colocalization of QTL for partial resistance with genes involved in major resistance has often been described on the chromosome scale (Marandel et al. 2009; Wisser 2005). However, the effects of allelic variants of a major gene on partial resistance have seldom been confirmed functionally or on a finemapping scale, except for some weak or defeated forms of NBLRR genes (Poland et al. 2009). Here, we identified a 151-kb interval containing RYMV-QTL1. Seventeen genes, including the four genes of the $R Y M V 2$ region, have been annotated in this interval. Any of them could be a candidate. However, the probability of observing colocalization by chance in a $151-\mathrm{kb}$ interval of a major gene and a nonfunctionally associated QTL is low, suggesting that the same gene probably controls both partial and high resistance. According to the Genevestigator reference expression database (Hruz et al. 2008), LOC_Os01g68970 gene, hereafter referred to as $O s C P R 5-1$, is expressed in various organs and at different developmental stages and deregulated by specific biotic and abiotic stresses. In addition, a 1.8-fold difference in $O s C P R 5-1$ expression is reported between three pairs of $O$. sativa indica/O. sativa japonica varieties, with a higher expression level in japonica varieties $(P=0.005$ for one pair; $P<$ 0.001 for two pairs), suggesting regulation differences between the two subspecies. We also noticed indel events between IR64 and Azucena sequences in the promotor $/ 5^{\prime}$-UTR region of CPR5-1. These indels might be associated with differences in regulation. If OsCPR5- 1 is actually involved in the establishment of defense mechanisms against RYMV, as hypothesized above, differences in OSCPR5-1 regulation may have a quantitative impact on the speed of defense mechanism establishment or intensity, resulting in differences in susceptibility level associated with the detection of RYMV-QTL1 effect. Similarly, a QTL for resistance against Magnaporthe grisea and Xanthomonas oryzae pv. oryzicola is supposed to result from differences in the promoter of an indole-3-acetic acid-amido synthatase involved in auxin metabolism (Fu et al. 2011).

Finally, our results suggest that $O s C P R 5-1$ may be a negative regulator of the defense response, similar to the mlo gene, whose effectiveness at the field level in barley resistance against mildew has not been denied for decades (Lyngkjær et al. 2000). OsCPR5-1 may be involved in partial or high resistance of rice against RYMV, depending on the allele. A null allele may completely prevent the virus cycle and lead to high resistance, while functional differences in the protein or regulation may quantitatively affect defense mechanisms and lead to partial resistance. Results obtained on $A$. thaliana cpr5 mutants suggest that $O s C P R 5$-1-mediated resistance may be efficient against several pathogens. Thus, in the next $R Y M V 2$ characterization steps, particular attention will be focused on the resistance of the Tog7291 accession to other pathogens such as M. grisea or $X$. oryzae, two major rice pathogens.

\section{MATERIALS AND METHODS}

\section{Plant accessions.}

Tog7291 (corresponding to International Risk Governance Council [IRGC] accession number 104589) and Tog5681 are O. glaberrima accessions resistant to RYMV. Tog7291 carries a resistance allele at the $R Y M V 2$ locus, while Tog5681 carries a susceptibility allele on $R Y M V 2$ but a resistance allele on RYMV1 (Albar et al 2006; Thiémélé et al. 2010). Azucena is a partially resistant $O$. sativa japonica variety and IR64 is a highly susceptible $O$. sativa indica variety. This study also involved a collection of rice accessions, including $100 \mathrm{O}$. 
barthii accessions from the Institut de Recherche pour le Développement (IRD) (formerly ORSTOM) rice collection and $261 O$. glaberrima and $56 O$. sativa varieties from indica and japonica subspecies. Among the $O$. glaberrima accessions, 30 and 140 were reported to be highly resistant and susceptible, respectively, to RYMV (Thiémélé et al. 2010). All the $O$. sativa accessions were susceptible or partially resistant. The resistance phenotype is unknown in $O$. barthii accessions. The resistance of some $O$. glaberrima accessions was analyzed in this study. Inoculation and symptom observation was performed as described in the following paragraph. ELISA tests were performed as described by Ndjiondjop and associates (1999) to estimate virus content in the plants.

\section{Populations and phenotypic evaluation for $R Y M V 2$ mapping.}

The first mapping step of $R Y M V 2$ was performed on an F2 intraspecific population derived from the Tog5681 $\times$ Tog7291 cross. Because both $R Y M V 1$ and $R Y M V 2$ are segregating in this population, this analysis involved only 156 informative F2 plants, nonhomozygous for the Tog5681 resistance allele on RYMV1 (i.e., for which resistance cannot be attributed to $R Y M V 1$.) An interspecific population derived from the Tog7291 $\times$ IR64 cross was developed for fine mapping of $R Y M V 2$. This population included $43 \mathrm{BC} 1 \mathrm{~F} 1$ plants obtained using Tog7291 as recurrent parent, $318 \mathrm{BC} 1 \mathrm{~F} 2$, and 3,470 $\mathrm{BC} 1 \mathrm{~F} 3$. These $\mathrm{BC} 1 \mathrm{~F} 2$ and $\mathrm{BC} 1 \mathrm{~F} 3$ plants were derived from $\mathrm{BC} 1 \mathrm{~F} 1$ and $\mathrm{BC} 1 \mathrm{~F} 2$ plants, respectively, that were heterozygous and nonrecombinant in the candidate region. Depending of the population, all the plants or only the recombinant plants were phenotyped, and resistance evaluation was performed either directly on the genotyped plants or on their progenies, as indicated in Table 4. RYMV resistance was assessed in glasshouse conditions on the basis of symptom intensity. IR64 and Tog7291 were used as susceptible and resistant controls, respectively. Inoculation was performed in the seedling stage, approximately 2 weeks after sowing, except for BC1F1 plants, for which inoculation was performed on cuttings. Plants were mechanically inoculated with an RYMV isolate from Burkina Faso (BF1), as described by Thiémélé and associates (2010). The presence of symptoms was checked twice per week for 3 weeks. Susceptible plants de- veloped clear yellow mottles 10 to 15 days postinoculation, whereas resistant plants had no symptoms at all.

\section{Populations and phenotypic evaluation for RYMV-QTL1 mapping.}

Fine mapping of RYMV-QTL1 involved advanced backcrossed lines. A recombinant inbred line from the IR64 $\times$ Azucena population carrying the IR64 allele at RYMV-QTL1 was selected (Boisnard et al. 2007) and backcrossed three times with Azucena. BC3F2 and BC3F3 plants were screened for recombination between RM431 and RM5310 markers. In order to reduce phenotypic variability, plants were also screened for the RM101 marker, tagging another major QTL for partial resistance located on chromosome 12 (Boisnard et al. 2007); only plants fixed for the IR64 allele on RM101 were selected for further experiments. For each $\mathrm{BC} 3 \mathrm{~F} 2$ recombinant, $\mathrm{BC} 3 \mathrm{~F} 3$ progenies fixed on both sides of the recombination were selfed; phenotypic evaluations were then performed on $\mathrm{BC} 3 \mathrm{~F} 4$ plants obtained from two to four BC3F3 plants having the same genotype on RYMV-QTL1 region. For BC3F3 recombinants, fixed $\mathrm{BC} 3 \mathrm{~F} 4$ progenies were selected and $\mathrm{BC} 3 \mathrm{~F} 5$ progenies were evaluated.

A first phenotyping step involved the 17 available recombinant families. Two different experiments were performed and seven genotypes were tested in both experiments for subsequent use as controls. Six repeats of five to seven plants and seven repeats of four plants per genotype were tested in experiments 1 and 2, respectively. Plants were mechanically inoculated 20 days after sowing with the BF1 isolate, and symptoms were scored on a 1-to-9 intensity scale (Thiémélé et al. 2010). Symptoms appeared 7 days postinoculation and became more difficult to estimate from 13 days postinoculation. Finally, two observations ( 8 and 11 days postinoculation) were retained for analysis because no significant difference (two-way analysis of variance, $P>0.05$ ) was observed between the two experiments on the control genotypes. The symptom intensity score, retained for analysis, is the mean of the two observations. A second phenotyping step involved only progenies of BC3F2-240, which was recombined between markers ID303300 and RM8236. Four BC3F3 progenies fixed for the IR64 allele on ID303300 and four $\mathrm{BC} 3 \mathrm{~F} 3$ progenies fixed for the Azucena allele were

Table 4. Number of plants genotyped and phenotyped from each population for RYMV2 mapping

\begin{tabular}{ll}
\hline Number of genotyped plants & Phenotyping conditions \\
\hline $\begin{array}{l}\text { Tog5681 } \times \text { Tog7291 population } \\
156 \text { F2 }\end{array}$ & Phenotyping on all the F2 plants, in the seedling stage \\
$\begin{array}{l}\text { Tog7291 } \\
\text { 43 BC1F1 } 64) \times \operatorname{Tog} 7291 \text { population }\end{array}$ & $\begin{array}{l}\text { Phenotyping on cuttings of the BC1F1 recombinant plants } \\
\text { 318 BC1F2, 3470 BC1F3 }\end{array}$ \\
$\begin{array}{c}\text { Phenotyping on the BC1F2 or BC1F3 recombinant plants when homozygous for Tog7291 allele on one side of } \\
\text { the recombination; phenotyping on the progenies of the other recombinant plants; in the seedling stage. }\end{array}$ \\
\hline
\end{tabular}

Table 5. Name and characteristics of markers designed for RYMV2 and RYMV-QTL1 fine mapping

\begin{tabular}{llll}
\hline Name & \multicolumn{1}{c}{ Forward primer } & \multicolumn{1}{c}{ Reverse primer $^{\text {Position }^{\mathbf{a}}}$} \\
\hline ID202770 & GGAGGCTGTAATGAGGGAAA & TCCAAAGCTTCAAACCCACT & $40,023,687$ \\
ID229216 & AATCGTCTGAGCCACCATCT & GTACAATAGCCGGCGACAAA & $40,050,051$ \\
ID232111 & TTAGATCTTCTGCCCCAACG & GAGCTTTTCTAGTTTCCATGGTG & $40,052,806$ \\
ID239585 & CCGGTCTCATGACCAAATG & TGTTACTGCCTCCTCGGAAT & $40,060,631$ \\
ID268747 & TTGCAATACGGTAAGATAAACTTCA & ACCACCATCTGCTGTTGCTA & $40,089,648$ \\
ID272679 & GAGTTCATCAGCTCCCGAAT & GCGTTCAGCACGAACAGAT & $40,093,721$ \\
Np40105633 & CCTCCTCCACGAAGTAGTGC & GCTACCTGGAGGCGATACTG & $40,106,676$ \\
ID302117 & CTGCAATGCAATCGAAGAAA & AGCCATAATCCATCGCCTTA & $40,123,027$ \\
ID303300 & AACAGGGGAAATCTGTGACG & GATCGATCGGCTTGTTTA & $40,124,102$ \\
ID421800 & TTTGTCATCCAAGGTGCTGA & GTTGAGGATGCAGCTTGTGA & $40,242,733$ \\
CAPS1 & CGAAGGACATTCCGAGAGG & CACGACGTCGCTTCGCCTCC & $40,073,472$ \\
CAPS2 & CAGACATGAACCCACACACC & AGCATCGTTTTCGTCCAAAC & $40,081,350$ \\
\hline
\end{tabular}

${ }^{a}$ Position refers to the position of the forward primer in the Nipponbare sequence (IRGSP-1.0). The marker names indicate if they correspond to simplesequence repeat (Np), indel (ID), or restriction site (cleaved amplified polymorphic sequence [CAPS]) polymorphism. 
selfed. The phenotype was evaluated on five repeats of four plants per $\mathrm{BC} 3 \mathrm{~F} 4$ progeny. Mechanical inoculation was performed as described for the first phenotyping step but the symptoms progressed more slowly. The symptom intensity score represents the mean of observations obtained 10,14, and 17 days postinoculation. Statistical analyses were performed on R software (R Development Core Team 2012).

\section{Genomic resources.}

The $O$. sativa subsp. japonica Nipponbare public sequence used in this study corresponded to the release Os-NipponbareReference-IRGSP-1.0 (Kawahara et al. 2013). Annotations referred to the MSU Rice Genome Annotation Project release 7.0 (Ouyang et al. 2007) for gene models and to the Rice Annotation Project Database (Sakai et al. 2013) for repeated sequences. Genes annotated as putative retrotransposon genes in the MSU project were not considered. The $O$. glaberrima genome assembly version 1, obtained on the CG14 accession, was downloaded from the Arizona Genomics Institute website. The homologous genes of interest from rice or other plant species were identified using the GreenPhyl database (Rouard et al. 2011).

\section{Molecular markers.}

A first set of markers used for RYMV2 or RYMV-QTL1 mapping was selected in a public rice SSR database (IRGSP 2005). Complementary SSR markers were identified with the MISA script (Thiel et al. 2003) on the Nipponbare sequence. In addition, indel markers were designed by comparing the Nipponbare sequence with the orthologous CG14 sequence based on dotplot alignments performed with Dotter software (Sonnhammer and Durbin 1995). Primers were designed with Primer3 (Untergasser et al. 2007) and found to amplify a single region on the Nipponbare genome using the Primer Blaster tool (Droc et al. 2009). Some markers designed to be polymorphic between Nipponbare and CG14 may be nonpolymorphic between CG14 and IR64. In addition, some of these markers proved to be polymorphic between IR64 and Azucena and, thus, useful for RYMV-QTL1 fine mapping. Information on the SSR and indel markers designed in this study is listed in Table 5.

A medium-throughput genotyping method was used for screening 1-week-old plants with two markers flanking RYMVQTL1 or RYMV2 candidate regions. A 1-mm-diameter leaf disc or $4 \mu \mathrm{l}$ of leaf extract $\left(1 \mathrm{~cm}^{2}\right.$ of leaf ground in $100 \mu$ l of water) were used as DNA template. PCR reactions were performed with the Terra PCR direct DNA polymerase kit (Takara Bio Europe/Clontech, Saint-Germain-en-Laye, France) in a final volume of $10 \mu \mathrm{l}$ containing $1 \times$ Terra PCR Direct Buffer, $0.3 \mu \mathrm{M}$ each primer, and $1.25 \mathrm{U}$ of Terra DNA polymerase. The cycling conditions were programmed for an initial denaturation at $98^{\circ} \mathrm{C}$ for $2 \mathrm{~min}$, followed by 30 cycles at $98^{\circ} \mathrm{C}$ for $10 \mathrm{~s}$ and $60^{\circ} \mathrm{C}$ for $15 \mathrm{~s}$, and a final extension of $1 \mathrm{~min}$ at $68^{\circ} \mathrm{C}$. Recombinant plants were further genotyped with additional markers under standard conditions. Total DNA was extracted from leaf tissue using the cetyltrimethylammonium bromide method (Murray and Thompson 1980). PCR was performed using GoTaq DNA polymerase (Promega Corp., Madison WI, USA) in a final volume of $15 \mu \mathrm{l}$ containing $20 \mathrm{ng}$ of template DNA, $1 \times$ GoTaq buffer, $1.5 \mathrm{mM} \mathrm{MgCl}_{2}, 0.2 \mathrm{mM}$ each dNTP, $0.25 \mu \mathrm{M}$ each primer, and $0.3 \mathrm{U}$ of GoTaq DNA polymerase. The cycling conditions were programmed for an initial 3-min cycle at $94^{\circ} \mathrm{C}$; followed by 30 cycles of $30 \mathrm{~s}$ at $94^{\circ} \mathrm{C}, 45 \mathrm{~s}$ at $55^{\circ} \mathrm{C}$, and $1 \mathrm{~min}$ at $72^{\circ} \mathrm{C}$; with a final extension of $5 \mathrm{~min}$ at $72^{\circ} \mathrm{C}$.

Sequences of the primers used for CAPS1 and CAPS2 amplification are given in Table 5. Because of the high GC content of the CAPS1 region, amplification was performed with the Terra PCR direct DNA polymerase kit (Takara Bio Europe/ Clontech) on leaf samples or DNA, as explained previously.
CAPS2 was amplified under standard conditions. After amplification, $10 \mu \mathrm{l}$ of buffer-restriction enzyme mixtures $(2 \mu \mathrm{l}$ of reaction buffer, $0.5 \mu \mathrm{l}$ of restriction enzyme $C f o \mathrm{I}, 0.2 \mu \mathrm{l}$ of bovine serum albumen, and $7.3 \mu \mathrm{l}$ of double-distilled $\mathrm{H}_{2} \mathrm{O}$ ) was added to $10 \mu \mathrm{l}$ of PCR product and incubated at $37^{\circ} \mathrm{C}$ for $2 \mathrm{~h}$.

Electrophoresis of PCR or digestion products was performed in agarose and revealed with ethidium bromide for polymorphism greater than $12 \mathrm{bp}$, in a Li-Cor sequencer 4600 (Li-Cor Biosciences, Lincoln, NE, U.S.A.) for polymorphisms between 6 and 12 bp using a M13 tag, as described by Albar and associates (2006).

\section{Sequence analysis.}

The 30-kb interval containing RYMV2 was sequenced in $\operatorname{Tog} 7291$, based on PCR amplification on genomic DNA extracted as described previously. Primers were designed on the region to amplify fragments of approximately $1 \mathrm{~kb}$. PCR fragments were amplified using GoTaq DNA Polymerase or the Terra PCR direct DNA Polymerase Kit for GC-rich regions, as described above, except that the elongation time was programmed at $1 \mathrm{~min} / \mathrm{kb}$. Sanger sequencing was subcontracted to Beckman Coulter Genomics (Takeley, U.K.). In the absence of mutation between Tog7291 and CG14, 1× coverage was considered sufficient, while mutations were confirmed on at least three different PCR products.

A complete genomic locus corresponding to the LOC_ Os01g68970 gene was sequenced in IR64 and Azucena, and partial cDNA was sequenced in IR64, Azucena, and CG14. RNA was extracted from leaves with the RNEasy Plant mini kit (Qiagen GmbH, Hamburg, Germany). Retrotranscription was performed using a poly-A primer and the ImProm2 reverse transcriptase (Promega Corp.) according to the manufacturer's recommendations. The 5'-CTCGCCTGCTTCTT CGAG-3' and 5'-AATCTGAGAGGTTGCGTGCT-3' forward primers and the 5'-CCGGCGAACCCAGTATGGTAAC-3' reverse primer were used to amplify transcripts corresponding to different gene models (Fig. 2). Amplification was performed using the classical protocol described previously. Amplification products obtained from genomic DNA were sequenced directly. Amplification products obtained from cDNA were cloned in PGEM-T Easy vector (Promega Corp.) according to the manufacturer's recommendations, and two different clones per accession were sent for sequencing.

Sequences were analyzed with Geneious software (v6.0.5; Biomatters, Auckland, New Zealand).

\section{ACKNOWLEDGMENTS}

This work was financially supported by the Agence Nationale de la Recherche (ANR, MOVIE Project). The Agence Universitaire de la Francophonie (AUF) provided a grant to D. Thiémélé, and the Département Soutien et Formation (DSF) of the IRD provided a grant to $\mathrm{O}$. Kolade. We thank M. Lorieux and F. Sabot for helpful discussions.

\section{LITERATURE CITED}

Albar, L., Lorieux, M., Ahmadi, N., Rimbault I, Pinel, A., Sy A, Fargette, D., and Ghesquière, A. 1998. Genetic basis and mapping of the resistance to Rice yellow mottle virus. I. QTLs identification and relationship between resistance and plant morphology. Theor. Appl. Genet. 97:1145-1154.

Albar, L., Bangratz-Reyser, M., Hébrard, E., Ndjiondjop, M.-N., Jones M, M., and Ghesquière, A. 2006. Mutations in the eIF(iso)4G translation initiation factor confer high resistance of rice to Rice yellow mottle virus. Plant J. 47:417-426.

Boisnard, A., Albar, L., Thiémélé, D., Rondeau, M., and Ghesquière, A. 2007. Evaluation of genes from $e I F 4 E$ and $e I F 4 G$ multigenic families as potential candidates for partial resistance QTLs to Rice yellow mottle virus in rice. Theor. Appl. Genet. 116:53-62.

Bowling, S. A., Clarke, J. D., Liu, Y., Klessig, D. F., and Dong, X. 1997. 
The cpr5 mutant of Arabidopsis expresses both NPR1-dependent and NPR1-independent resistance. Plant Cell 9:1573-1584.

Brininstool, G., Kasili, R., Simmons, L. A., Kirik, V., Hülskamp, M., and Larkin, J. C. 2008. Constitutive expressor of pathogenesis-related genes 5 affects cell wall biogenesis and trichome development. BMC Plant Biol. 8:58.

Brizard, J. P., Carapito, C., Delalande, F., Van Dorsselaer, A., and Brugidou, C. 2006. Proteome analysis of plant-virus interactome: Comprehensive data for virus multiplication inside their hosts. Mol. Cell. Proteomics 5:2279-2297.

Büschges, R., Hollricher, K., Panstruga, R., Simons, G., Wolter, M., Frijters, A., van Daelen, R., van der Lee, T., Diergaarde, P., Groenendijk, J., Töpsch, S., Vos, P., Salamini, F., and Schulze-Lefert, P. 1997. The barley Mlo gene: A novel control element of plant pathogen resistance. Cell 88:695-705.

Cosson, P., Sofer, L., Le, Q. H., Leger, V., Schurdi-Levraud, V., Whitham, S. A., Yamamoto, M. L., Gopalan, S., Le Gall, O., Candresse, T., Carrington, J. C., and Revers, F. 2010. RTM3, which controls long-distance movement of potyviruses, is a member of a new plant gene family encoding a meprin and TRAF homology domain-containing protein. Plant Physiol. 154:222-232.

Cournoyer, P., and Dineskumar, S. P. 2011. NB-LRR Immune receptors in plant virus defence. Pages 149-176 in: Recent Advances in Plant Virology. C. Caranta, M. A. Aranda, M. Tepfer, and J. J. Lopez-Moya, eds. Caister Academic Press, Norfolk, U.K.

Decroocq, V., Sicard, O., Alamillo, J. M., Lansac, M., Eyquard, J. P., Garcia, J. A., Candresse, T., Le Gall, O., and Revers, F. 2006. Multiple resistance traits control Plum pox virus infection in Arabidopsis thaliana. Mol. Plant-Microbe Interact. 19:541-549.

Di Carli, M., Villani, M. E., Bianco, L., Lombardi, R., Perrotta, G., Benvenuto, E., and Donini, M. 2010. Proteomic analysis of the plantvirus interaction in Cucumber mosaic virus (CMV) resistant transgenic tomato. J. Proteome Res. 9:5684-5697.

Dodds, P. N., and Rathjen, J. P. 2010. Plant immunity: Towards an integrated view of plant-pathogen interactions. Nat. Rev. Genet. 11:539-548.

Droc, G., Périn, C., Fromentin, S., and Larmande, P. 2009. OryGenesDB 2008 update: Database interoperability for functional genomics of rice. Nucleic Acids Res. 37:D992-D995.

Fu, J., Liu, H., Li, Y., Yu, H., Li, X., Xiao J, and Wang, S. 2011. Manipulating broad-spectrum disease resistance by suppressing pathogeninduced auxin accumulation in rice. Plant Physiol. 155:589-602.

Fujisaki, K., Iwahashi, F., Kaido, M., Okuno, T., and Mise, K. 2009. Genetic analysis of a host determination mechanism of bromoviruses in Arabidopsis thaliana. Virus Res. 140:103-111.

Gomez, P., Rodríguez-Hernández, A., Moury, B., and Aranda, M. 2009. Genetic resistance for the sustainable control of plant virus diseases: Breeding, mechanisms and durability. Eur. J. Plant Pathol. 125:1-22.

Gonzalez-Ibeas, D., Canizares, J., and Aranda, M. A. 2012. Microarray analysis shows that recessive resistance to Watermelon mosaic virus in melon is associated with the induction of defense response genes. Mol. Plant-Microbe Interact. 25:107-118.

Hruz, T., Laule, O., Szabo, G., Wessendorp, F., Bleuler, S., Oertle, L., Widmayer, P., Gruissem, W., and Zimmermann, P. 2008. Genevestigator v3: A reference expression database for the meta-analysis of transcriptomes. Adv. Bioinf. 2008:1-5.

IRGSP. 2005. The map-based sequence of the rice genome. Nature 436:793-800.

Jing, H.-C., and Dijkwel, P. P. 2008. CPR5: A Jack of all trades in plants. Plant Signal. Behav. 3:562-563

Jing, H.-C., Sturre, M. J. G., Hille, J., and Dijkwel, P. P. 2002. Arabidopsis onset of leaf death mutants identify a regulatory pathway controlling leaf senescence. Plant J. 32:51-63.

Kawahara, Y., La Bastide, de, M., Hamilton, J. P., Kanamori, H., McCombie, W. R., Ouyang, S., Schwartz, D. C., Tanaka, T., Wu, J., Zhou, S., Childs, K. L., Davidson, R. M., Lin, L., Quesada-Ocampo, L., Vaillancourt, B., Sakai, H., Lee, S. S., Kim, S., Numa, H., Itoh, T., Buell, C. R., and Matsumoto, T. 2013. Improvement of the Oryza sativa Nipponbare reference genome using next generation sequence and optical map data. Rice 6:4.

Khetarpal, R. K., Maisonneuve, B., Maury, Y., Chalhoub, B., Dinant, S., Lecoq, H., Varma, A., Hadidi, A., Khetarpal, R. K., and Koganezawa, H. 1998. Breeding for resistance to plant viruses. Pages 14-32 in: Plant Viruses Disease Control. A. Hadidi, R. K. Khetarpal, and H. Koganezawa, eds. American Phytopathological Society, St. Paul, MN.

Kirik, V., Bouyer, D., Schöbinger, U., Bechtold, N., Herzog, M., Bonneville, J. M., and Hülskamp, M. 2001. CPR5 is involved in cell proliferation and cell death control and encodes a novel transmembrane protein. Curr. Biol. 11:1891-1895.

Kouassi, N., N'Guessan, P., Albar, L., Fauquet, C., and Brugidou, C. 2005. Distribution and characterization of Rice yellow mottle virus: A threat to
African farmers. Plant Dis. 89:124-133.

Lee, J.-H., Muhsin, M., Atienza, G. A., Kwak, D.-Y., Kim, S.-M., De Leon, T. B., Angeles, E. R., Coloquio, E., Kondoh, H., Satoh, K., Cabunagan, R. C., Cabauatan, P. Q., Kikuchi, S., Leung, H., and Choi, I.-R. 2010. Single nucleotide polymorphisms in a gene for translation initiation factor (eIF4G) of rice (Oryza sativa) associated with resistance to Rice tungro spherical virus. Mol. Plant-Microbe Interact. 23:29-38.

Le Gall, O., Aranda, M. A., and Caranta, C. 2011. Plant resistance to viruses mediated by translation initiation factors. Pages 177-194 in: Recent Advances in Plant Virology. C. Caranta, M. A. Aranda, M. Tepfer, and J. J. Lopez-Moya, eds. Caister Academic Press, Norfolk, U.K.

Love, A. J., Laval, V., Geri, C., Laird, J., Tomos, A. D., Hooks, M. A., and Milner, J. J. 2007. Components of Arabidopsis defense- and ethylenesignaling pathways regulate susceptibility to Cauliflower mosaic virus by restricting long-distance movement. Mol. Plant-Microbe Interact. 20:659-670.

Lyngkjær, M. F., Newton, A. C., Atzema, J. L., and Baker, S. J. 2000. The barley mlo-gene: An important powdery mildew resistance source. Agronomie 20:745-756.

Marandel, G., Pascal, T., Candresse, T., and Decroocq, V. 2009. Quantitative resistance to Plum pox virus in Prunus davidiana P1908 linked to components of the eukaryotic translation initiation complex. Plant Pathol. 58:425-435.

Mlotshwa, S., Pruss, G. J., and Vance, V. 2008. Small RNAs in viral infection and host defense. Trends Plant Sci. 13:375-382.

Murray, M. G., and Thompson, W. F. 1980. Rapid isolation of high molecular weight plant DNA. Nucleic Acids Res. 8:4321-4325.

Ndjiondjop, M.-N., Albar, L., Fargette, D., Fauquet C, and Ghesquière, A. 1999. The genetic basis of high resistance to rice yellow mottle virus (RYMV) in cultivars of two cultivated rice species. Plant Dis. 83:931-935.

Ouyang, S., Zhu, W., Hamilton, J., Lin, H., Campbell, M., Childs, K., Thibaud-Nissen, F., Malek, R.L., Lee, Y., Zheng, L., Orvis, J., Haas, B., Wortman, J., and Buell, C. R. 2007. The TIGR Rice Genome Annotation Resource: Improvements and new features. Nucleic Acids Res. 35:D883-D887.

Pavan, S., Jacobsen, E., Visser, R. G. F., and Bai, Y. 2009. Loss of susceptibility as a novel breeding strategy for durable and broad-spectrum resistance. Mol. Breed. 25:1-12.

Poland, J. A., Balint-Kurti, P. J., Wisser, R. J., Pratt, R. C., and Nelson, R. J. 2009. Shades of gray: The world of quantitative disease resistance. Trends Plant Sci. 14:21-29.

R Development Core Team 2012. R: A Language and Environment for Statistical Computing. R Foundation for Statistical Computing, Vienna.

Rouard, M., Guignon, V., Aluome, C., Laporte, M.-A., Droc, G., Walde, C., Zmasek, C. M., and Périn, C. 2011. GreenPhylDB v2.0: Comparative and functional genomics in plants. Nucleic Acids Res. 39:D1095-D1102.

Sakai, H., Lee, S. S., Tanaka, T., Numa, H., Kim, J., Kawahara, Y., Wakimoto, H., Yang, C.-C., Iwamoto, M., Abe, T., Yamada, Y., Muto, A., Inokuchi, H., Ikemura, T., Matsumoto, T., Sasaki, T., and Itoh, T. 2013. Rice Annotation Project Database (RAP-DB): An integrative and interactive database for rice genomics. Plant Cell Physiol. 54:e6.

Siré, C., and Brugidou, C. 2002. Les virus du riz: État des lieux, impact et méthodes de lutte. Virologie 6:431-443.

Sonnhammer, E. L., and Durbin, R. 1995. A dot-matrix program with dynamic threshold control suited for genomic DNA and protein sequence analysis. Gene 167:GC1-10.

Thiel, T., Michalek, W., Varshney, R. K., and Graner, A. 2003. Exploiting EST databases for the development and characterization of genederived SSR-markers in barley (Hordeum vulgare L.). Theor. Appl. Genet. 106:411-422.

Thiémélé, D., Boisnard, A., Ndjiondjop, M.-N., Chéron, S., Séré, Y., Aké, S., Ghesquière, A., and Albar, L. 2010. Identification of a second major resistance gene to Rice yellow mottle virus, RYMV2, in the African cultivated rice species, O. glaberrima. Theor. Appl. Genet. 121:169-179.

Untergasser, A., Nijveen, H., Rao, X., Bisseling, T., Geurts, R., and Leunissen, J. A. M. 2007. Primer3Plus, an enhanced web interface to Primer3. Nucleic Acids Res. 35:W71-4.

Wang, A., and Krishnaswamy, S. 2012. Eukaryotic translation initiation factor 4E-mediated recessive resistance to plant viruses and its utility in crop improvement. Mol. Plant Pathol., 13:795-803.

Wisser, R. J. 2005. Identification and characterization of regions of the rice genome associated with broad-spectrum, quantitative disease resistance. Genetics 169:2277-2293.

Yoshida, S., Ito, M., Nishida, I., and Watanabe, A. 2002. Identification of a novel gene $H Y S 1 / C P R 5$ that has a repressive role in the induction of leaf senescence and pathogen-defence responses in Arabidopsis thaliana. Plant J. 29:427-437.

Yoshii, M., Shimizu, T., Yamazaki, M., Higashi, T., Miyao, A., Hirochika, H., and Omura, T. 2009. Disruption of a novel gene for a NAC-domain protein in rice confers resistance to Rice dwarf virus. Plant J. 57:615-625. 